OPEN

SUBJECT AREAS:

PHYSICS

ENGINEERING

Received

15 October 2014

Accepted

7 January 2015

Published

2 February 2015

Correspondence and requests for materials should be addressed to T.J.C. (tjcui@seu.edu.

\section{Broadband Frequency-Selective Spoof Surface Plasmon Polaritons on Ultrathin Metallic Structure}

\author{
Jia Yuan Yin ${ }^{1}$, Jian Ren ${ }^{2}$, Hao Chi Zhang' ${ }^{1}$ Bai Cao Pan' \& Tie Jun Cui ${ }^{1,3}$
}

\begin{abstract}
'State Key Laboratory of Millimeter Waves, School of Information Science and Engineering, Southeast University, Nanjing 210096 , China, ${ }^{2}$ Science and Technology on Antenna and Microwave Laboratory, Xidian University, Xi'an 710071, China, ${ }^{3}$ Cooperative Innovation Center of Terahertz Science, University of Electronic Science and Technology, Chengdu 61 1731, China.
\end{abstract}

We propose an ultrathin metallic structure to produce frequency-selective spoof surface plasmon polaritons (SPPs) in the microwave and terahertz frequencies. Designed on a thin dielectric substrate, the ultrathin metallic structure is composed of two oppositely oriented single-side corrugated strips, which are coupled to two double-side corrugated strips. The structure is fed by a traditional coplanar waveguide (CPW). To make a smooth conversion between the spatial modes in CPW and SPP modes, two transition sections are also designed. We fabricate and measure the frequency-selective spoof SPP structure in microwave frequencies. The measurement results show that the reflection coefficient is less than $-10 \mathrm{~dB}$ with the transmission loss around $1.5 \mathrm{~dB}$ in the selective frequency band from 7 to $10 \mathrm{GHz}$, which are in good agreements with numerical simulations. The proposed structure can be used as an SPP filter with good performance of low loss, high transmission, and wide bandwidth in the selective frequency band.

ecause of the negative permittivity behavior of metals at visible frequencies, surface plasmon polaritons (SPPs) are propagating on the interface of metal and dielectric with exponential decay in the direction vertical to the interface ${ }^{1,2}$. However, the metals will behave like perfectly electric conductors (PECs) instead of plasmas with negative permittivity when the frequency is reduced to the terahertz and microwave bands, which do not support the existence of SPPs. In order to realize SPPs in such lower frequencies, plasmonic metamaterials have been proposed to produce the so-called spoof $\mathrm{SPPs}^{3-14}$. The plasmonic metamaterials are usually formed by one- or two-dimensional periodical textures on metal surfaces. Among them, there had been some valuable works on subwavelength corrugated structures which can be used to transmit spoof SPPs ${ }^{910-13}$. Ref. 9 showed how the dispersion relation of SPPs propagating along a perfectly conducting wire can be tailored by corrugating its surface with a periodic array of radial grooves. With this method, highly localized SPPs can be sustained in the terahertz region of the electromagnetic spectrum. The realization of planar plasmonic terahertz guided-wave devices using periodically performed metal films was described ${ }^{11}$. The experimental results showed that the propagating terahertz radiation was well confined in both the in-plane and out-of-plane axes. The other way of guiding terahertz waves along corrugated V-grooves was proposed in Ref. 12. This kind of structure presents longer propagation lengths and lower bending losses, making it the excellent candidate for guiding terahertz waves on planar surfaces. The corrugated metallic wedges can also route the terahertz waves, which featured the subwavelength confinement in the transverse plane ${ }^{13}$. Based on the proposed wedge waveguides, the frequency selective focusing and slow terahertz radiation have also been demonstrated ${ }^{13}$. More recently, an ultrathin corrugated metallic strip with subwavelength width has been proposed using a competitive structure, which can support and propagate conformal SPPs on arbitrarily curved surfaces ${ }^{14}$. As the plasmonic metamaterials are advancing at a rapid pace, they are considered as a solid avenue for real applications.

One of the advantages of plasmonic metamaterials is the simple structure, which offers convenience for fabrications. Many efforts have been made to generate highly confined spoof SPP waves ${ }^{3-14}$ and design functional devices $^{3-14}$ in the microwave and terahertz frequencies. However, a problem has emerged as the plasmonic metamaterials are developed: the SPP functional components and circuits need to be connected to the conventional microwave and terahertz circuits, which are commonly composed of two-conductor transmission lines like microstrips and coplanar waveguides (CPWs). That is to say, it is very important to convert the spatial waves supported by the traditional transmission lines to SPP modes supported by the plasmonic structures with high efficiency. In the past, the conversion between single- and two-conductor transmission lines has been studied ${ }^{15-18}$. 


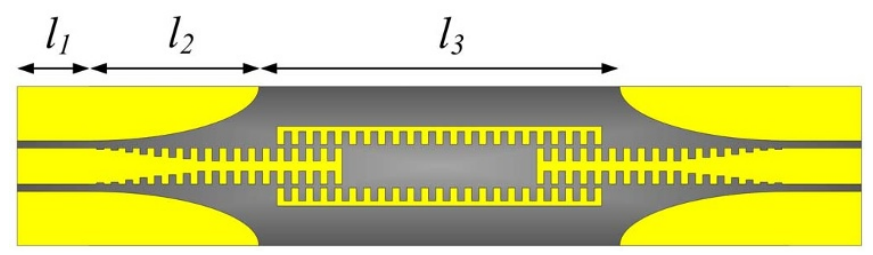

Figure 1 Schematic picture of the ultrathin metallic frequency-selective structure, in which the yellow part is metal (modeled as copper) and the dark gray part is thin dielectric substrate. The length of each part is $l_{1}=10 \mathrm{~mm}, l_{2}=60 \mathrm{~mm}$, and $l_{3}=123 \mathrm{~mm}$.

Recently, a broadband and efficient conversion from spatially guided waves to SPPs in the microwave frequency has been proposed ${ }^{19}$, in which gradient corrugation grooves and flaring ground are presented to match both the momentum and impedance. In Ref. 20, the broadband transition between microstrip line and conformal surface plasmon waveguide was also presented.

Based on the successful developments of mode conversions, plasmonic functional devices catch more and more attentions. As a key component, the filter plays a very important role in the wireless communication, radar, and other systems. To design an SPP filter, a bulky structure was presented using periodic subwavelength metallic Domino array ${ }^{21}$. Recently a method to control the rejections of SPPs has been proposed using metamaterial particles $^{22}$. Meanwhile, a double-grating plasmonic waveguide and band-pass filter have been proposed in Ref. 23, employing a stepped slot line to couple electromagnetic energies from the microstrip. However, the above SPP filters are either bulky or performance limited.

In this article, we propose an ultrathin metallic structure printed on a thin dielectric substrate to produce the frequencyselective spoof SPPs in the microwave and terahertz frequencies, which is composed of two oppositely oriented single-side corrugated strips coupled to two double-side corrugated strips. In order to feed the structure and extract the transmitted SPPs by traditional CPWs, two transition sections are also designed for smooth conversion between spatial modes in CPWs and SPPs. This compact structure can be used as a good SPP filter. We fabricate and measure the SPP filter in the microwave frequency, and the measurement results show that the reflection coefficient is less than $-10 \mathrm{~dB}$ and the transmission loss is around $1.5 \mathrm{~dB}$ in the selective frequency range from 7 to $10 \mathrm{GHz}$. Hence the proposed SPP filter is compact but has good performance of low loss, high transmission, and wide band, which is important to produce the plasmonic integrated circuits in microwave and terahertz frequencies in the near future.

\section{Results}

As shown in Fig. 1, the proposed frequency-selective spoof SPP structure is designed using ultrathin metallic strips, which are printed on a thin and flexible dielectric film. In the current design in the microwave frequency, the thickness of metal (copper) film is $0.018 \mathrm{~mm}$, while the thickness of dielectric substrate is $d=0.05 \mathrm{~mm}$, which correspond to $6 / 10000$ and $17 / 10000$ wavelengths at $10 \mathrm{GHz}$, respectively. For designs and theoretical characterizations, the parameters of this particular structure are accurately retrieved as: $l_{1}=10 \mathrm{~mm}, l_{2}=60 \mathrm{~mm}$, and $l_{3}=123 \mathrm{~mm}$. As demonstrated in Fig. 1, the proposed structure is connected to two CPW sections with $50-\Omega$ impedance to feed energies and receive the transmitted SPP signals.

The whole structure of the SPP frequency-selective filter is composed of three parts, as illustrated in Fig. 2. The first part is CPW (see Fig. 2(a)) for the purposes to feed or receive electromagnetic fields. To achieve the 50- $\Omega$ impedance, the CPW parameters are chosen as $w=15 \mathrm{~mm}, g=0.55 \mathrm{~mm}$, and $H=10 \mathrm{~mm}$. The second part is a transition section between CPW and the SPP waveguide, a double-side corrugated metallic strip. The details of the transition design have been demonstrated in Ref. 19, according to which the transition structure consists of a flaring ground and gradient grooves, as shown in Fig. 2(b). The flaring ground is designed to match the impedance between CPW and SPP waveguide, while the gradient grooves are used to match the momentum. The designed dimensions of the transition part are given as: $h_{1}=0.5 \mathrm{~mm}, h_{2}=1 \mathrm{~mm}, h_{3}=1.5 \mathrm{~mm}$, $h_{4}=2 \mathrm{~mm}, h_{5}=2.5 \mathrm{~mm}, h_{6}=3 \mathrm{~mm}, h_{7}=3.5 \mathrm{~mm}$, and $h_{8}=4 \mathrm{~mm}$. The curvature of the flaring ground is $k=0.2$. The third part is the main structure constructed by a double-side corrugated strip and two single-side corrugated strips, as illustrated in Fig. 2(c), in which the period, width, and depth of metallic grooves are chosen as $p=5 \mathrm{~mm}$, $a=3 \mathrm{~mm}$, and $h=4 \mathrm{~mm}$. The two coupled single-side corrugated strips with opposite orientation directions are aimed to realize the frequency-selective characteristics.

Figure 3 gives the dispersion curves of the single-side and doubleside corrugated metallic strips. We observe that the cut-off frequency of the double-side is a little bit lower than that of single-side, at around $11 \mathrm{GHz}$. Based on the concept of microwave network, the coupling part can be described by a four-port network ${ }^{24}$, as illustrated in the central diagram in Fig. 4. Because of the symmetry of the structure, the analysis of coupling is only taken half into account. For the four ports 1, 2, 3, and 4 marked in Fig. 4, we get the following relationships

$\left[\begin{array}{l}b_{1} \\ b_{2} \\ b_{3} \\ b_{4}\end{array}\right]=e^{-j \beta z}\left[\begin{array}{cccc}0 & \cos (\kappa z) & -i \sin (\kappa z) & 0 \\ \cos (\kappa z) & 0 & 0 & -i \sin (\kappa z) \\ -i \sin (\kappa z) & 0 & 0 & \cos (\kappa z) \\ 0 & -i \sin (\kappa z) & \cos (\kappa z) & 0\end{array}\right]\left[\begin{array}{l}a_{1} \\ a_{2} \\ a_{3} \\ a_{4}\end{array}\right](1)$

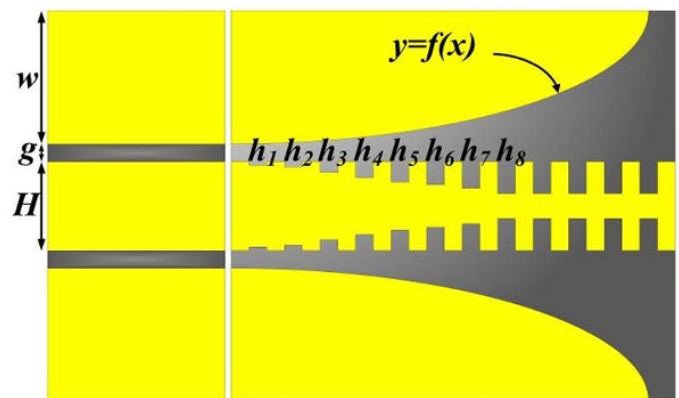

(a) (b)

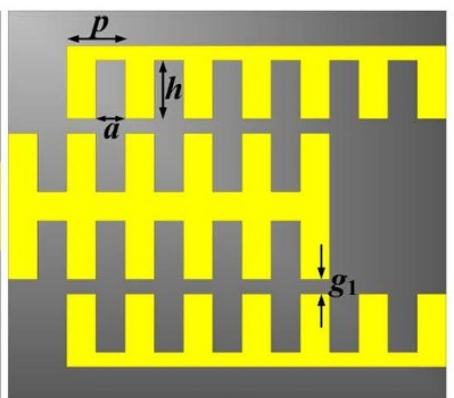

(c)

Figure $2 \mid$ The configuration of the frequency-selective SPP structure, which is composed of three parts. (a) The CPW section, in which $w=15$ mm, $g=0.55 \mathrm{~mm}$, and $H=10 \mathrm{~mm}$. (b) The transition with flaring ground and gradient grooves, in which $h_{1}=0.5 \mathrm{~mm}, h_{2}=1 \mathrm{~mm}, h_{3}=1.5 \mathrm{~mm}, h_{4}=2 \mathrm{~mm}$, $h_{5}=2.5 \mathrm{~mm}, h_{6}=3 \mathrm{~mm}, h_{7}=3.5 \mathrm{~mm}$, and $h_{8}=4 \mathrm{~mm}$. The curvature of the flaring ground is $k=0.2$. (c) The corrugated metallic strips, in which $p=5 \mathrm{~mm}, a=3 \mathrm{~mm}, h=4 \mathrm{~mm}$, and $g_{1}=1 \mathrm{~mm}$. 


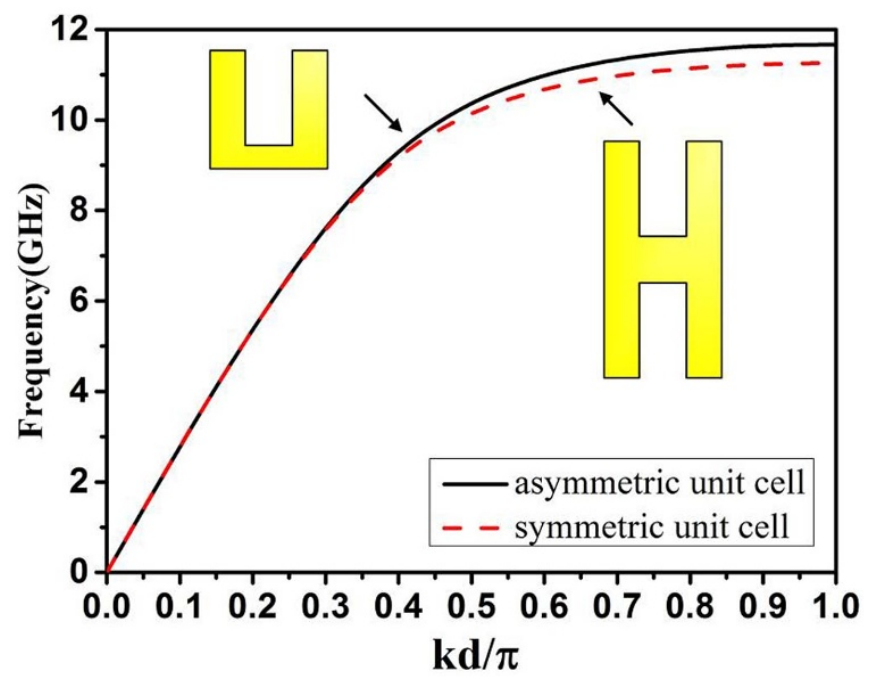

Figure 3 The dispersion curves of single-side and double-side corrugated metallic strips.

$$
\left[\begin{array}{l}
b_{2} \\
b_{4}
\end{array}\right]=E\left[\begin{array}{l}
a_{2} \\
a_{4}
\end{array}\right]
$$

where $\kappa$ is the coupling coefficient, and $z$ is the length of coupling part. Then the S scattering parameter matrix of the coupling part is deduced as

$$
S=\left[\begin{array}{cc}
\frac{\cos ^{2}(\kappa z)}{e^{2 j \beta z}+\sin ^{2}(\kappa z)} & -\frac{2 i \sin (\kappa z) e^{j \beta z}}{e^{2 j \beta z}+\sin ^{2}(\kappa z)} \\
-\frac{2 i \sin (\kappa z) e^{j \beta z}}{e^{2 j \beta z}+\sin ^{2}(\kappa z)} & \frac{\cos ^{2}(\kappa z)}{e^{2 j \beta z}+\sin ^{2}(\kappa z)}
\end{array}\right]
$$

From the S-parameter matrix, we notice that the transmission coefficient $\left(S_{21}\right)$ can be zero under specific circumstances, implying that the SPP waves cannot propagate through the coupling part.

To study the control of frequency-selective bands by the two single-side corrugated metallic strips, two parameters are chosen to investigate the influence of the coupling part using numerical simulations. One parameter is the gap $g_{1}$ between two corrugated metallic strips, while the other parameter is the length of the coupling strip. Fig. 5(a) illustrates the total transmission coefficients $\left(\mathrm{S}_{21}\right)$ of the whole structure with different gaps, in which the dielectric constant and tangent loss of the substrate are set as 2.65 and 0.01 , respectively. It is obvious that SPPs are only allowed propagations under certain frequency bands. As the gap is smaller, the coupling strength becomes larger with a slight redshift of bandwidth. The influence of the coupling length is demonstrated in Fig. 5(b), in which different numbers $(n)$ of unit cells are used in the coupling part. We note that the coupling length has significant impact on the selective frequency bandwidth. For longer coupling length $(n=4)$, the selective frequency has a larger bandwidth from 7 to $10 \mathrm{GHz}$; for shorter coupling length $(n=2)$, the selective frequency has a smaller bandwidth from 9.5 to $10.5 \mathrm{GHz}$. We observe that the black line in Fig. 5 drops

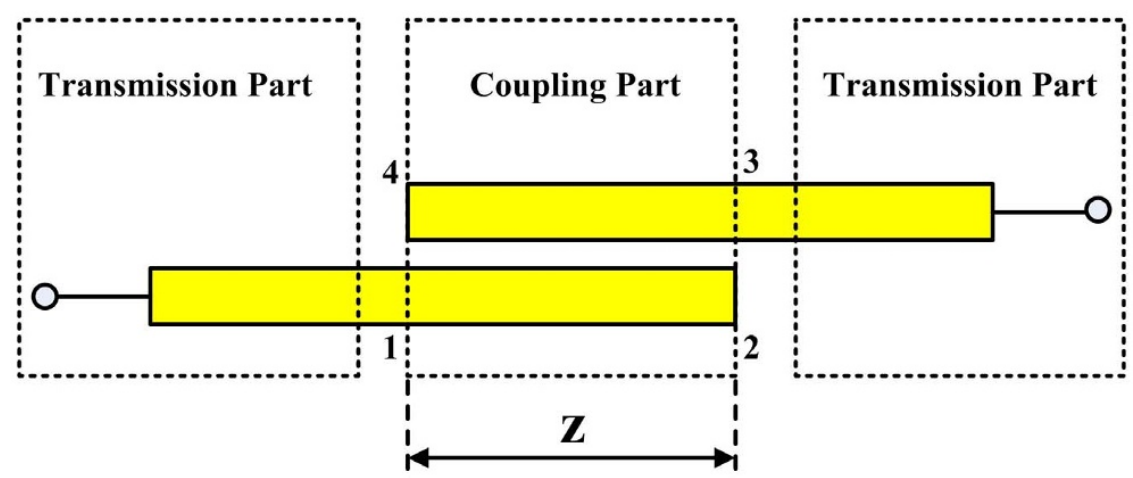

Figure 4 | Schematic of the coupling part of the proposed frequency-selective structure.

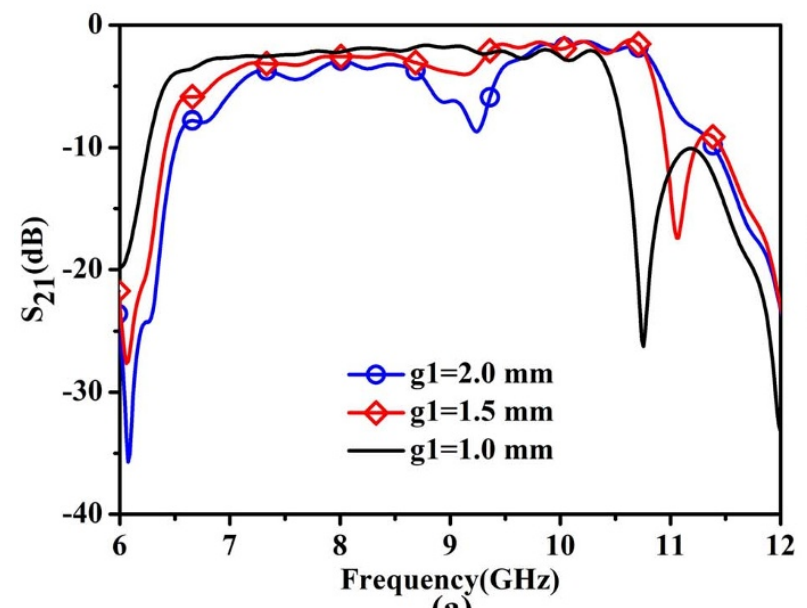

(a)

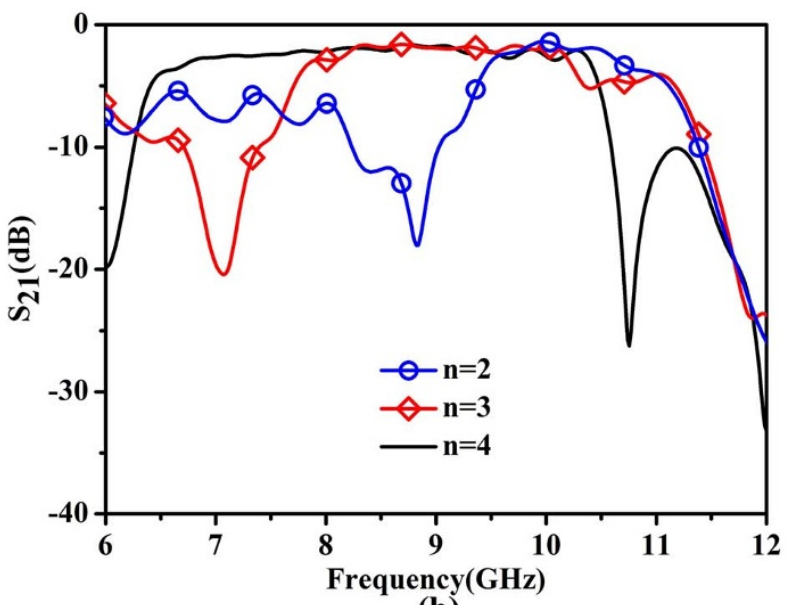

(b)

Figure $5 \mid$ The simulated transmission coefficients $\left(S_{21}\right)$ with different strip gaps $\left(g_{1}\right)$ and numbers of coupling unit cells $(n)$. (a) The black, red, and blue lines correspond to $g_{1}=1 \mathrm{~mm}, 1.5 \mathrm{~mm}$, and $2 \mathrm{~mm}$, respectively, in which $n=4$ is fixed. (b) The black, red, and blue lines correspond to $n=4$, 3, and 2, respectively, in which $g_{1}=1 \mathrm{~mm}$ is fixed. 


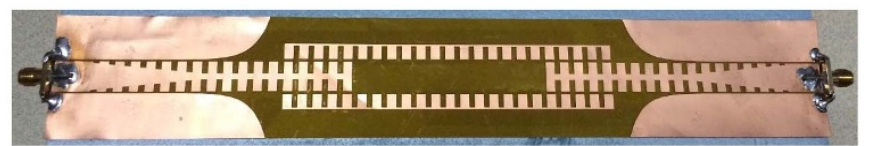

Figure 6 The photograph of the fabricated frequency-selective SPP structure, which is printed on a flexible dielectric film.

quickly at about $10.8 \mathrm{GHz}$, which implies a transmission zero isolating the stop band from the operating band. Then the black line rises again at about $11 \mathrm{GHz}$. This phenomenon can be explained by the combined effect of resonance and cutoff frequency. Owing to the transmission zero, the periodic frequency response curve is supposed to appear with crest and trough alternately. When the cutoff frequency is between the crest and trough, the peak emerges. In fact, the dispersion relation of the coupling part is relevant to the transmission spectrum. Since the dispersion curve characterizes the relation between the propagation constant and frequency, and the coupling strength is also related to the frequency, we can tune the dispersion relation of the coupling part through the coupling strength, and furthermore tune the transmission spectrum.

In experiments in the microwave frequency, we fabricate a sample of the frequency selective structure, in which the gap between two adjacent corrugated strips is set as $g_{1}=1 \mathrm{~mm}$, the number of coupling units is chosen as $n=4$, and the dielectric constant and loss tangent of the substrate are the same as those in simulations, as illustrated in Fig. 6. The measured and simulated results of the $S$ parameters (reflection coefficients $S_{11}$ and transmission coefficients $S_{21}$ ) are shown in Fig. 7, which have good agreements to each other. We notice that the reflection coefficients $\left(S_{11}\right)$ are less than $-10 \mathrm{~dB}$ from 7 to $10 \mathrm{GHz}$, which verifies the good impedance and momentum matching from CPW to SPP waveguide. As illustrated in Fig. 7(b), the transmission coefficients indicate good frequency-selective property of the proposed structure. The transmission loss is only around $1.5 \mathrm{~dB}$ in the selective frequency band.

In order to demonstrate the frequency-selective characteristics clearly, we also give the near-field distributions above the structure at different frequencies. Figure 8 presents the simulated near electric fields on an observation plane, which is $4 \mathrm{~mm}$ above the proposed structure. At $6 \mathrm{GHz}$ and $11 \mathrm{GHz}$, the electric-field distributions are shown in Figs. 8(a) and 8(d), respectively. It is obviously that the energy cannot propagate through the coupling structure since such frequencies are beyond the selected frequency band. On the other hand, at $7 \mathrm{GHz}$ and $9 \mathrm{GHz}$, which are within the selected frequency band, excellent mode conversions and energy propagations are

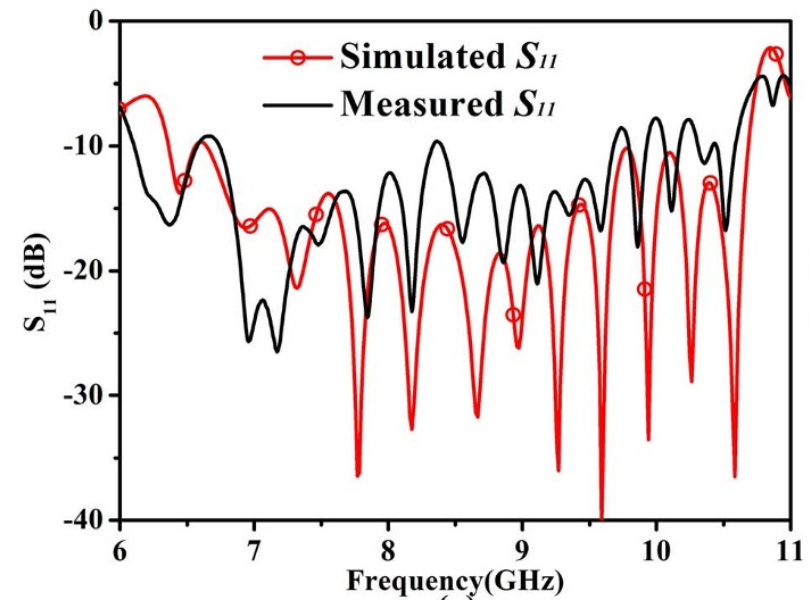

(a)

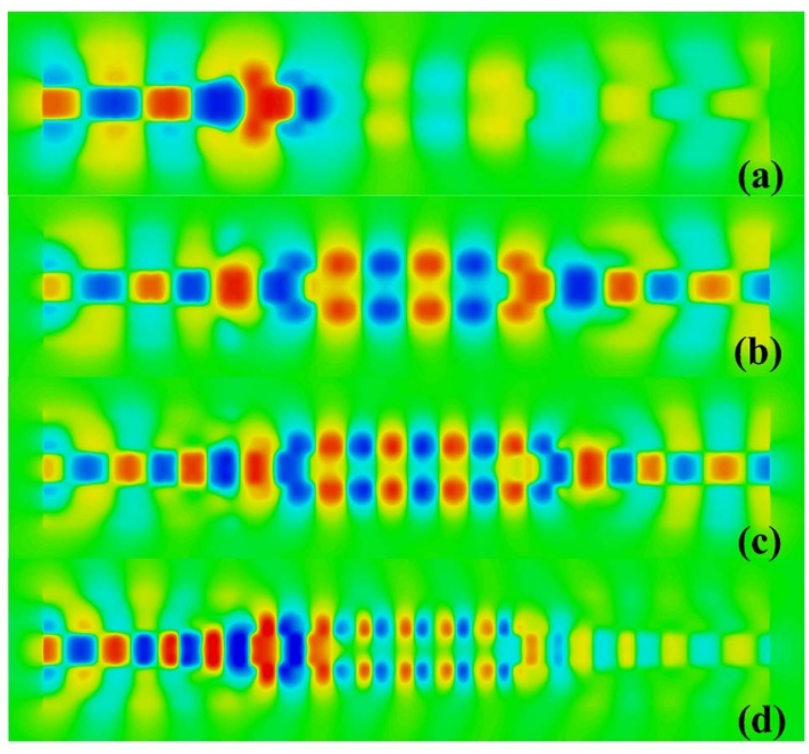

Figure $8 \mid$ The simulated near electric-field distributions. (a) The frequency is $6 \mathrm{GHz}$, which is beyond the selected frequency band. (b) The frequency is $7 \mathrm{GHz}$, which is within the selected frequency band. (c) The frequency is $9 \mathrm{GHz}$, which is within the selected frequency band.

(d) The frequency is $11 \mathrm{GHz}$, which is beyond the selected frequency band.

clearly observed, as displayed in Figs. 8(b) and 8(c). Figure 9 provides the relevant measurement results of the near electric fields, which are in very good agreements with the simulation results. As discussed in Figs. 8 and 9, small reflections and high transmissions of SPPs are realized in the selected frequency band using the proposed structure, which can be used as an SPP band-pass filter. The good measurement results suggest that the proposed SPP filter should be a promising device for the plasmonic integrated circuits in the microwave frequencies.

\section{Discussion}

In this article, we have proposed a compact and ultrathin structure to produce frequency selective spoof SPPs in the microwave frequencies. In particular, we presented four coupled corrugated metallic strips to reach the frequency-selective properties. Besides the coupled structure, we also proposed two transition sections to covert the SPP modes to spatial transverse electromagnetic modes, so as to connect

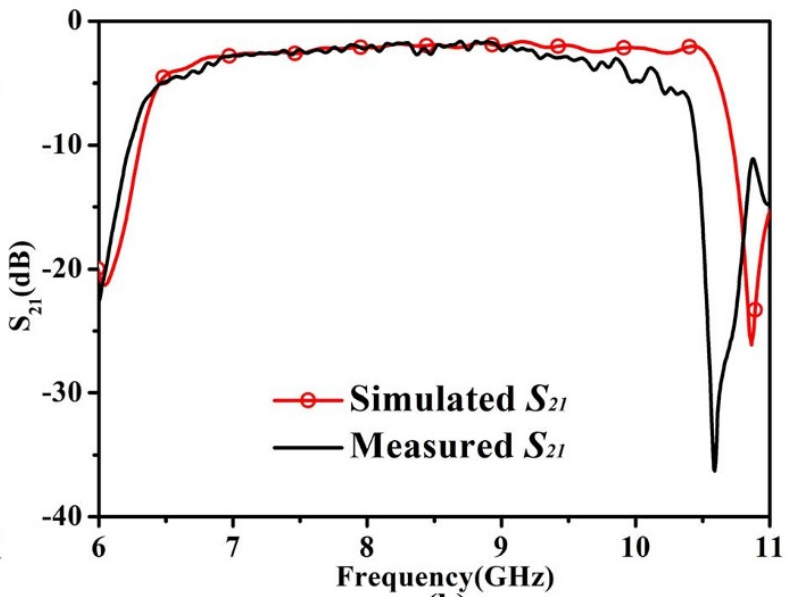

(b)

Figure $7 \mid$ The simulation and measurement results of $S$ parameters, in which the red lines are simulations and the black lines are measurements. (a) The reflection coefficients $\left(S_{11}\right)$. (b) The transmission coefficients $\left(S_{21}\right)$. 
8. Navarro-Cía, M. et al. Broadband spoof plasmons and subwavelength electromagnetic energy confinement on ultrathin metafilms. Opt. Exp. 17, 18184-18195 (2009).

9. Maier, S. A., Andrews, S. R., Martin-Moreno, L. \& Garcia-Vidal, F. Terahertz surface plasmon-polariton propagation and focusing on periodically corrugated metal wires. Phys. Rev. Lett. 97, 176805 (2006).

10. Shah, C. M. et al. Design and analysis of a metasurface for supporting spoof surface plasmon polaritons. Optoelectronic and Microelectronic Materials \& Devices (COMMAD), 2012 Conference on., Melbourne, VIC. Piscataway:IEEE. (2012, Dec. 12th-14th).

1. Zhu, W., Agrawal, A. \& Nahata, A. Planar plasmonic terahertz guided-wave devices. Opt. Exp. 16, 6216-6226 (2008).

12. Fernandez-Dominguez, A., Moreno, E., Martin-Moreno, L. \& Garcia-Vidal, F. Guiding terahertz waves along subwavelength channels. Phys. Rev. B 79, 233104 (2009).

13. Fernández-Domínguez, A., Moreno, E., Martin-Moreno, L. \& Garcia-Vidal, F. Terahertz wedge plasmon polaritons. Opt. Lett. 34, 2063-2065 (2009).

14. Shen, X., Cui, T. J., Martin-Cano, D. \& Garcia-Vidal, F. J. Conformal surface plasmons propagating on ultrathin and flexible films. Proc. Natl. Acad. Sci. 110, 40-45 (2013).

Figure 9| The measured near electric-field distributions. (a) The frequency is $6 \mathrm{GHz}$, which is beyond the selected frequency band. (b) The frequency is $7 \mathrm{GHz}$, which is within the selected frequency band. (c) The frequency is $9 \mathrm{GHz}$, which is within the selected frequency band.

(d) The frequency is $11 \mathrm{GHz}$, which is beyond the selected frequency band.

with CPWs smoothly in feeding and receiving electromagnetic fields. We made numerical simulations and experiments to validate our design. Both the simulated and measured S parameters and nearelectric-field results demonstrate that the proposed structure has high transmissions (with insertion loss of $1.5 \mathrm{~dB}$ ) and low reflections (below $-10 \mathrm{~dB}$ ) in the selected frequency band from 7 to $10 \mathrm{GHz}$. Such performance makes the proposed structure be a good SPP band-pass filter. The proposed filter may be further used in multilayer structures in the future for higher integrations. We remark that the method can be extended to the terahertz frequencies to realize plasmonic functional devices and integrated circuits.

\section{Methods}

Numerical simulations are performed by the commercial software, CST Microwave Studio. The experimental structure is fabricated using a $0.05-\mathrm{mm}$ thin dielectric film with dielectric constant 2.65 and tangent loss 0.01 , respectively. The thickness of metal (copper) film is $0.018 \mathrm{~mm}$. We use Agilent Vector Network Analyzer to measure the $S$ parameters (i.e., the reflection coefficients $\mathrm{S}_{11}$ and transmission coefficients $S_{21}$ ) of the fabricated sample. The near electric-filed distributions are measured by a home-made near-field scanning system, in which the probe is set as $2 \mathrm{~mm}$ above the fabricated sample.

1. Harvey, A. Periodic and guiding structures at microwave frequencies. IEEE T. Microw. Theory 8, 30-61 (1960).

2. Barnes, W. L., Dereux, A. \& Ebbesen, T. W. Surface plasmon subwavelength optics. Nature 424, 824-830 (2003).

3. Pendry, J., Martin-Moreno, L. \& Garcia-Vidal, F. Mimicking surface plasmons with structured surfaces. Science 305, 847-848 (2004).

4. Hibbins, A. P., Evans, B. R. \& Sambles, J. R. Experimental verification of designer surface plasmons. Science 308, 670-672 (2005).

5. Garcia-Vidal, F., Martin-Moreno, L. \& Pendry, J. Surfaces with holes in them: new plasmonic metamaterials. J. Opt. A- Pure.Appl. Op. 7, S97 (2005).

6. Zhou, Y. J., Jiang, Q. \& Cui, T. J. Bidirectional bending splitter of designer surface plasmons. Appl. Phys. Lett. 99, 111904 (2011).

7. Gan, Q., Fu, Z., Ding, Y. J. \& Bartoli, F. J. Ultrawide-bandwidth slow-light system based on THz plasmonic graded metallic grating structures. Phys. Rev. Lett. 100, $256803(2008)$
15. Treizebré, A., Akalin, T. \& Bocquet, B. Planar excitation of Goubau transmission lines for THz bioMEMS. Microw. Wirel. Co. Lett., IEEE 15, 886-888 (2005).

16. Goubau, G. Open wire lines. IEEE T.Microw. Theory 4, 197-200 (1956).

17. Akalin, T., Treizebré, A. \& Bocquet, B. Single-wire transmission lines at terahertz frequencies. IEEE T.Microw. Theory 54, 2762-2767 (2006).

18. Chen, W.-C., Mock, J., Smith, D., Akalin, T. \& Padilla, W. Controlling gigahertz and terahertz surface electromagnetic waves with metamaterial resonators. Phys. Rev. X 1, 021016 (2011).

19. Ma, H. F., Shen, X., Cheng, Q., Jiang, W. X. \& Cui, T. J. Broadband and highefficiency conversion from guided waves to spoof surface plasmon polaritons. Laser Photonics Rev. 8, 146-151 (2014).

20. Liao, Z., Zhao, J., Pan, B. C., Shen, X. P. \& Cui, T. J. Broadband transition between microstrip line and conformal surface plasmon waveguide. J. Appl. Phys 47, 315103 (2014).

21. Wu, J. et al. Bandpass filter based on low frequency spoof surface plasmon polaritons. Electron. Lett. 48, 269-270 (2012).

22. Pan, B. C., Liao, Z., Zhao, J. \& Cui, T. J. Controlling rejections of spoof surface plasmon polaritons using metamaterial particles. Opt. Exp. 22, 13940-13950 (2014).

23. Gao, X., Zhou, L., Liao, Z., Ma, H. F. \& Cui, T. J. An ultra-wideband surface plasmonic filter in microwave frequency. Appl. Phys. Lett. 104, 191603 (2014).

24. Haus, H. \& Huang, W. Coupled-mode theory. P. IEEE 79, 1505-1518 (1991).

\section{Acknowledgments}

This work was supported in part by the National Science Foundation of China (60990320, $60990324,61171024,61171026$, and 61138001), in part by the National High Tech (863) Projects (2012AA030402 and 2011AA010202), and in part by the 111 Project (111-2-05)

\section{Author contributions}

J.Y.Y. and J.R. conceived the idea. J.Y.Y. did the simulations and optimization. H.C.Z. did the theoretical analysis. J.Y.Y., J.R. and B.C.P performed the measurements. J.Y.Y. and T.J.C. wrote the manuscript based on the input from all authors. All authors contributed to the discussion.

\section{Additional information}

Competing financial interests: The authors declare no competing financial interests.

How to cite this article: Yin, J.Y., Ren, J., Zhang, H.C., Pan, B.C. \& Cui, T.J. Broadband Frequency-Selective Spoof Surface Plasmon Polaritons on Ultrathin Metallic Structure. Sci. Rep. 5, 8165; DOI:10.1038/srep08165 (2015).

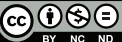

This work is licensed under a Creative Commons Attribution-NonCommercialNoDerivs 4.0 International License. The images or other third party material in this article are included in the article's Creative Commons license, unless indicated otherwise in the credit line; if the material is not included under the Creative Commons license, users will need to obtain permission from the license holder in order to reproduce the material. To view a copy of this license, visit http:// creativecommons.org/licenses/by-nc-nd/4.0/ 